\title{
CORRECTION
}

\section{Correction to: Volume 39 Issue 3, September 2021}

Published online: 13 November 2021

(c) Springer Science+Business Media, LLC, part of Springer Nature 2021

Correction to: Plant Molecular Biology Reporter (2021) 39(3) https://link.springer.com/journal/11105/volumes-and-issues/39-3

The original version of this issue unfortunately had incorrect cover year information. It was written as 2016 and it has been corrected to 2021 .

The original articles of the issue have been corrected.

Springer Nature, NY, USA.

Publisher's Note Springer Nature remains neutral with regard to jurisdictional claims in published maps and institutional affiliations.

The original articles can be found online at https://doi.org/10. 1007/s11105-020-01239-6 and https://doi.org/10.1007/s11105020-01263-6 and https://doi.org/10.1007/s11105-020-01264-5 and https://doi.org/10.1007/s11105-020-01266-3 and https://doi.org/ 10.1007/s11105-020-01267-2 and https://doi.org/10.1007/s11105020-01268-1 and https://doi.org/10.1007/s11105-020-01269-0 and https://doi.org/10.1007/s11105-020-01270-7 and https://doi.org/ 10.1007/s11105-020-01272-5 and https://doi.org/10.1007/s11105020-01273-4 and https://doi.org/10.1007/s11105-020-01274-3 and https://doi.org/10.1007/s11105-020-01275-2 and https://doi.org/ 10.1007/s11105-020-01278-z and https://doi.org/10.1007/s11105021-01279-6 and https://doi.org/10.1007/s11105-021-01280-z. 